\title{
Propuesta de RUteo de vehículos con flota HETEROGÉNEA Y VENTANAS DE TIEMPO (HFVRPTW) APLICADA A UNA COMERCIALIZADORA PYME DE LA CIUDAD DE CALI
}

\author{
Approach of Routing of VeHICLES With heterogenEOUS FLEET AND TIME \\ WINDOWS (HFVRPTW) APPLIED TO A RETAILER OF THE CITY OF CALI
}

Jairo Arboleda Zúñiga*

John Alexander Gaviria-Gómez"*

John Alexander Álvarez-Romero

Recibido: 15 de diciembre de 2017

Aceptado: 18 de mayo de 2018

\section{Resumen}

Este artículo estudia el problema del ruteo de vehículos con flota heterogénea y ventanas de tiempo (HFVRPTW) para dar solución a un problema real aplicado a una pequeña comercializadora de productos alimenticos de la ciudad de Cali que debe atender un conjunto de clientes con demandas conocidas y que tiene que usar un intervalo de tiempo predefinido por cada cliente. El modelo fue desarrollado en tres fases: caracterización del proceso actual de programación de ruta y distribución, adaptación del modelo matemático y solución del modelo por medio del método heurístico de ahorros, el cual se adapta de manera eficiente al problema. Los resultados evidencian mejoras sustanciales relacionadas con la disminución en el número de rutas de hasta el 40 \% para el vehículo tipo NHR, en la distancia recorrida del 50 \% para el vehículo Dmax y en los costos totales de transporte para los dos tipos de vehículos.

Palabras clave: ruteo de vehículos, flota heterogénea, ventanas de tiempo, método de los ahorros.

\section{Abstract}

This article addresses the problem of vehicle with heterogeneous fleet routing and windows of time (HFVRPTW) to solve a real problem applied to a small marketer of products food additives in the city of Cali, which must meet a set of customers with popular demands, where each client must be attended within a predefined time interval. The model was developed in three phases: the characterization of the current programming process route and distribution, the adaptation of the mathematical model and solution of the model by means of heuristic method of savings, which adapts efficiently to the problem and results with substantial improvements related to the

\footnotetext{
* Ingeniero industrial, magíster en Ingeniería Industrial. Investigador del Grupo-IDEAS, docente de la Facultad de Ingeniería, Universidad Santiago de Cali, Santiago de Cali, Colombia. http://orcid.org/0000-0003-4045-9396. jarboledaz@usc.edu.co

${ }^{* \star}$ Ingeniero Industrial, Universidad Santiago de Cali, Santiago de Cali, Colombia. http://orcid.org/0000-0002-29774005. john.gaviria00@usc.edu.co

*** Ingeniero Industrial, Universidad Santiago de Cali, Santiago de Cali. Colombia. http://orcid.org/0000-0001-60958825. jhonn.alvarez03@usc.edu.co
} 
number of routes of up to $40 \%$ for vehicle type NHR, decrease in the distance of $50 \%$ for the Dmax vehicle and decrease in total costs of transportation for 2 types of vehicles.

Keywords: vehicle routing, heterogeneous fleet, time windows, savings method.

\section{INTRODUCCIÓN}

Para desarrollar la competitividad en el mercado del siglo XXI, "la logística industrial es usada por las compañías con el fin de generar ventajas competitivas” (Rocha, González y Orjuela, 2011, p. 35). La logística industrial permite a una organización, en la operación de sus funciones logísticas, buscar la manera en que el costo de sus rutas siempre sea el mínimo. Al respecto, Toth y Vigo (2000, citado en Gonzales y Gonzales, 2006) afirman que "el problema de distribuir productos desde cierto depósito a sus usuarios finales juega un papel central en la gestión de algunos sistemas logísticos y su adecuada planificación puede significar considerables ahorros”. Por su parte, Sepúlveda, Escobar y Adarme-Jaimes (2014) consideran que "planear y controlar las actividades de distribución implica necesariamente la toma de decisiones sobre el ruteo de vehículos (VRP)" (p. 224).

Esta toma de decisiones por parte de las pequeñas y medianas empresas (PyME) de nuestro país se realiza principalmente de forma intuitiva, debido a que los "recursos que se invierten para planear y controlar las actividades de distribución son limitados; asimismo la robustez e innovación de sus recursos técnicos y tecnológicos son bajos" (Sepúlveda et ál., 2014, p. 223). Por esta razón se obtienen resultados insatisfactorios, que se relacionan con aumentos en los costos operativos de la flota de vehículos y la disminución en la productividad de los mismos; factores que afectan la rentabilidad y competitividad de las empresas.

La comercializadora objeto de estudio, al igual que las demás PyME colombianas, soporta de manera informal la toma de decisiones (principalmente, las decisiones relacionadas con las actividades de distribución), ya que no cuenta con las herramientas tecnológicas ni con el personal capacitado para planear y controlar de una manera más efectiva la programación y el diseño de rutas. Estos factores han ocasionado una serie de efectos negativos para los intereses de la comercializadora, relacionados con mayores distancias y tiempos de recorrido al momento de realizarlas entregas, incumplimiento en algunos casos y subutilización de la flota de vehículos. Esto último ocurre principalmente porque la comercializadora utiliza solo el $40 \%$ del volumen total del furgón, lo que en peso (kg) representa únicamente un $15 \%$, es decir, solo una sexta parte del peso total del camión NHR.

El objetivo de esta investigación es elaborar una propuesta de ruteo de vehículos, en la que se pretende establecer secuencias de visitas para cada cliente que permitan reducir costos de transporte. Aquí se consideran factores claves como flota heterogénea y ventanas de tiempo (o HFVRPTW, por sus siglas en inglés), además de un único depósito y múltiples clientes, presentes en la distribución de productos de consumo alimenticio de una pequeña empresa comercializadora colombiana.

Este artículo se estructura en cuatro secciones: la sección 1 propone una revisión de la literatura sobre las características del problema y heurística; la sección 2 trata sobre las tres fases metodológicas para la solución del problema: caracterización del proceso actual de programación de ruta y distribución, adaptación del modelo matemático y método de ahorros con ventanas de tiempo; la sección 3 presenta los resultados, y la sección 4 expone las conclusiones y propone perspectivas para una investigación futura. 


\section{REVISIÓN DE LITERATURA}

\section{HFVRPTW}

Según Paraskevopoulos, Repoussis, Tarantilis, Loannou y Prastacos (2008), el HFVRPTW implica el diseño de un conjunto de rutas de costo mínimo (cada ruta inicia y finaliza en el depósito), en el que se utiliza una flota heterogénea de vehículos con costos fijos y variables, para atender un conjunto de clientes con demandas conocidas; cada cliente se visita solo una vez (en un intervalo de tiempo determinado) por un vehículo establecido, de manera que el total de la demanda de una ruta no debe exceder la capacidad del vehículo asignado para esta labor. De acuerdo con Paraskevopoulos et ál. (2008), existen dos tipologías del HFVRPTW: el FSMVRPTW (o problema de ruteo de vehículos con tamaño de flota y mezcla $)^{1}$ implica varios tipos de vehículos de disponibilidad ilimitada, cuyo objetivo es determinar la composición óptima de la flota con unos costes de distribución mínimos globales (la suma de los costes fijos y variables). Por su parte, el HFFVRPTW (o problema de ruteo de vehículos con flota fija heterogénea) ${ }^{2}$ generaliza el FSMVRPTW, limitando el número de vehículos disponibles de cada tipo que se pueden desplegar.

El HFVRPTW es un problema NP-hard (Golden et ál.,1984) porque generaliza el problema de ruteo de vehículos con ventanas de tiempo (VRPTW), en las que cada vehículo tiene capacidades y costos diferentes, además de que se añade una ventana de tiempo en la cual un cliente puede ser atendido y en la que "se debe asignar el tipo de vehículo que atenderá a un conjunto de clientes y la ruta a seguir para satisfacer sus demandas" (Puenayán, Londoño, Escobar y Linfati, 2014, p. 83).

El-Sherbeny (2010) propone algoritmos exactos para la metodología HFVRPTW. Belfiore y Yoshizaki (2013) realizan una revisión de la literatura sobre HFVRPTW en la que muestran el tipo de variantes que puede adoptar este problema y los métodos heurísticos propuestos para solucionarlo.

Salhi, Wassan y Hajarat (2013) plantean un HFVRPTW en el que se aplica un método se solución heurística basado en el problema de partición de conjuntos (SPP). La heurística propuesta mejoró la calidad de las soluciones, pero requirió mucho tiempo de CPU.

Hiermann, Puchinger, Ropke y Hartl (2015) proponen un problema de tamaño de la flota eléctrica y la mezcla del enrutamiento del vehículo con las ventanas de tiempo y las estaciones de recarga (EFSMFTW). Este problema se resuelve proponiendo una heurística híbrida que combina una búsqueda adaptativa de grandes vecindarios con un procedimiento de búsqueda y etiquetado interno, lo que resuelve con facilidad pequeños problemas, pero presenta errores en la solución de problemas con mayor cantidad de nodos.

La investigación de Repoussis y Tarantilis (2010) expone un nuevo enfoque de solución de programación de memoria adaptativa (AMP) para el problema de tamaño de la flota y enrutamiento del vehículo de mezcla con ventanas de tiempo (FSMVRPTW).

Toro, Escobar y Granada (2016) desarrollan una revisión sobre problema de ruteo de vehículos, en la cual se presenta un enfoque de ventanas de tiempo y flota heterogénea por aparte, en la cual se plantean "modelos matemáticos y estrategias de solución que puedan ser aplicadas en organizaciones que consideren dentro de sus objetivos una operación eficiente y sustentable" (pp.362-363)

En la investigación de Domínguez-Martín, Rodríguez-Martín y Salazar-González (2018) se expone la solución al problema de que las rutas de los vehículos comienzan en un depósito y terminan en el otro, mientras que los conductores realizan rutas circulares que se abren y finalizan

\footnotetext{
${ }^{1}$ Término introducido por Golden, Assad, Levy y Gheysens (1984).

2 Término introducido por Liu y Shen (1999).
} 
en el mismo depósito y sus rutas no pueden exceder una duración determinada. En este estudio se desarrollaron procedimientos de separación para estas desigualdades válidas, implementando un algoritmo exacto de ramificación y corte que es capaz de resolver instancias con hasta 30 nodos en características individuales.

Ciancio, Laganá, y Vocaturo (2018) tratan la solución a un problema ruteo vehicular general con capacidad mixta con ventanas de tiempo (MCGRPTW) en un problema de enrutamiento de nodo equivalente en un gráfico dirigido. Los autores resuelven el problema equivalente mediante el uso de un algoritmo de precio de corte y corte. Igualmente, presentan experimentos computacionales sobre instancias derivadas del problema de enrutamiento de arco capacitado con ventanas de tiempo y del problema de enrutamiento general con capacidades mixtas en un tiempo de solución mayor a 6 horas.

\section{Heurísticas}

Una revisión de la literatura relacionada para los métodos heurísticos aplicados al VRP ha sido propuesta por Oliveira (2004). El autor muestra el tipo de variantes que puede adoptar estos métodos heurísticos planteados para solucionar un TPS y que posteriormente fueron adaptados a soluciones para VRP. Al respecto, según lo propone Salomon (1987):

En la heurística de barrido los clusters se forman girando una semirrecta con origen en el depósito e incorporando los clientes "barridos" por dicha semirrecta hasta que se viole la restricción de capacidad, este proceso tiene dos etapas: primero, las paradas se asignan a los vehículos, y luego se determina la secuencia de las paradas dentro de las rutas. (p. 258)

La heurística de "asignar primero y rutear después" (cluster first-route second) se da en dos fases:

Primero se busca generar grupos de clientes, también llamados clusters, que estarán en una misma ruta en la solución final. Luego, para cada cluster se crea una ruta que visite a todos sus clientes. Las restricciones de capacidad son consideradas en la primera etapa, asegurando que la demanda total de cada cluster no supere la capacidad del vehículo. (Olivera, 2004, p. 16)

Igualmente, la heurística de inserción:

Crea soluciones mediante sucesivas inserciones de clientes en las rutas, es decir, en cada iteración se tiene una solución parcial cuyas rutas solo visitan un subconjunto de los clientes y luego se selecciona un cliente no visitado para insertarlo en la última ruta creada. (Rocha, González y Orjuela, 2011, p. 46)

Por otra parte,

El objetivo del método de ahorros es minimizar la distancia total viajada por todos los vehículos y minimizar indirectamente el número de vehículos necesarios para atender todas las paradas. La lógica del método es empezar con la interacción de un vehículo que cubre cada nodo cliente y que regresa al nodo depósito. (Ballou, 2004, p.263)

Esto suministra la mayor distancia a tener en cuenta en el problema del diseño de ruta. Posteriormente:

Se combinan dos paradas en la misma ruta para que el vehículo pueda eliminarse y la distancia del viaje se reduzca. Para determinarlas paradas que se van a combinar en una ruta, se calcula la distancia 
ahorrada, antes y después de la combinación, la distancia ahorrada al combinar dos puntos (A y B) que no estén de otra manera en una ruta con cualquier otra parada, el resultado es un valor de ahorro, este cálculo se realiza para todos los pares de paradas, el par de paradas con el valor de ahorro más grande se selecciona para la combinación. (Ballou, 2004, p. 263)

\section{Metodología}

En esta sección se presenta el diseño metodológico para resolver el problema planteado, el cual consta de la combinación de tres fases: caracterización del proceso actual de programación de ruta y distribución, formulación del modelo matemático y aplicación del método de ahorros con ventanas de tiempo y flota heterogénea.

\section{Fase1}

La primera fase comprende la caracterización de la situación actual de la comercializadora. La figura 1 divide en tres las características principales identificadas del problema en el que se encuentran las rutas, los clientes y el medio de transporte, considerando los principales rasgos identificados para este problema.

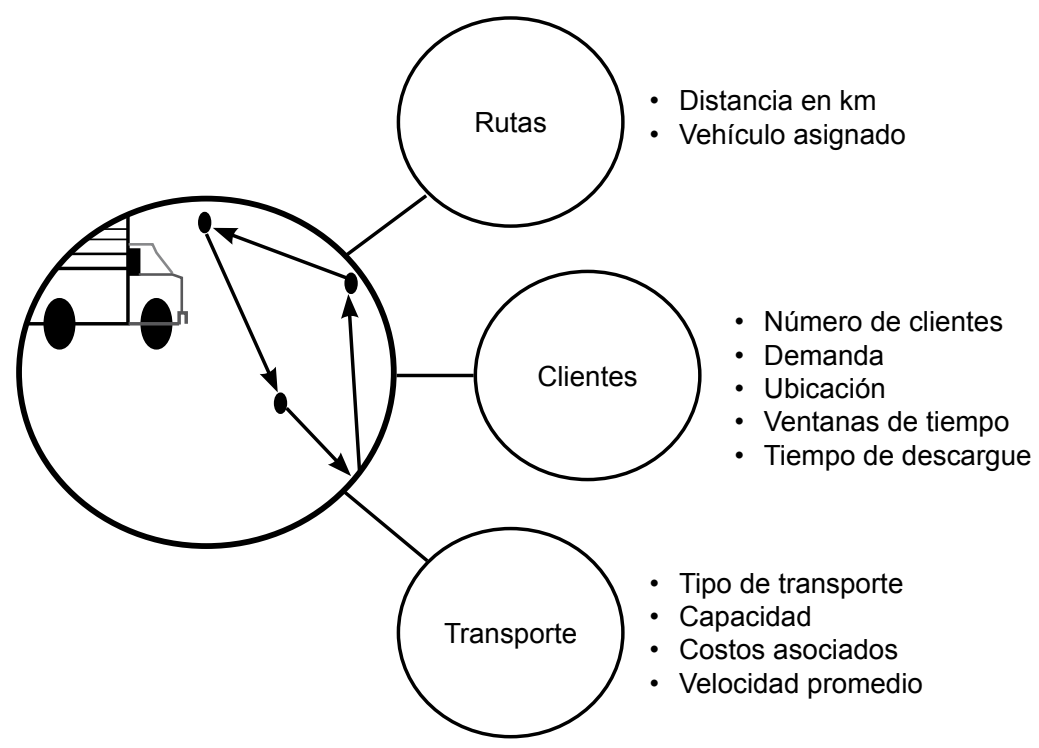

Figura 1. Caracterización de la situación actual

\section{Fase 2}

A partir de los resultados obtenidos previamente en la fase 1, se realizó una búsqueda en la literatura de los principales modelos propuestos para el HFVRPTW. Se examinaron más de cuarenta modelos, entre artículos de carácter científico y libros. Los artículos de investigación indexados fueron consultados en las bases de datos Science Direct y ProQuest.

\section{Fase 3}

Esta fase comprende la selección del método heurístico a partir del uso de una técnica multicriterio AHP, con la cual a partir de la aplicación se implementó el método heurístico de ahorros para 
resolver el problema HFVRPTW de la comercializadora. La aplicación de la técnica multicriterio y el método son desarrollados en el siguiente apartado.

La figura 2 describe la aplicación del método heurístico de ahorros. Este método busca a partir de la aplicación del método de ahorros encontrar la mejor ruta, por medio de la cual se empieza con la construcción de rutas con el cálculo de los ahorros entre los diferentes puntos (se tienen en cuenta los mayores ahorros en distancia para empezar a formar las rutas). Una vez se tiene la tabla se pasa a construir las diferentes rutas uniendo los diferentes puntos, revisando si es factible la unión. En este método no se tiene en cuenta la capacidad del vehículo y las ventanas de tiempo en la que se comparan dos nodos.

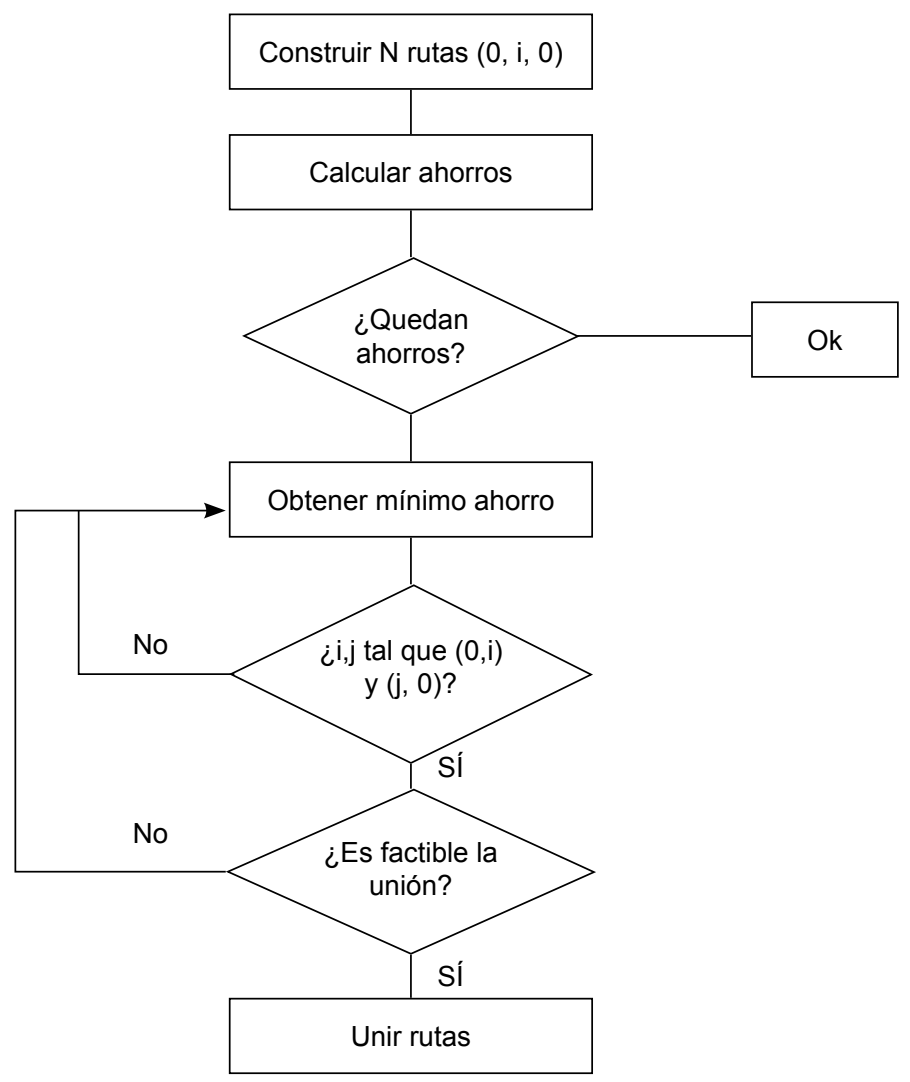

Figura 2. Aplicación método de ahorros

La figura 3 describe la aplicación del método heurístico de ahorros teniendo en cuenta las ventanas de tiempo y la flota heterogénea, en la cual se busca, a partir de la aplicación del método de ahorros, encontrar el mejor ruteo a la comercializadora. Este método empieza con la construcción de rutas a partir del cálculo de los ahorros entre los diferentes puntos. Una vez se tiene el cálculo de los ahorros, se empieza la construcción de las diferentes rutas, revisando si es factible la unión; en este caso se considera la capacidad de los diferentes vehículos y qué ventanas de tiempo tiene cada punto, lo cual determina en gran parte qué nodo se asigna a las diferentes rutas. Para este caso en particular, en el apartado de ventanas de tiempo se tuvieron en cuenta cada selección con el fin de que en esta ventana se cumpliera en todo momento hasta llegar a un tiempo de $8 \mathrm{~h}$ promedio, dado que este era el tiempo límite de entrega de la empresa y que la capacidad del vehículo no fuera a ser mayor. 


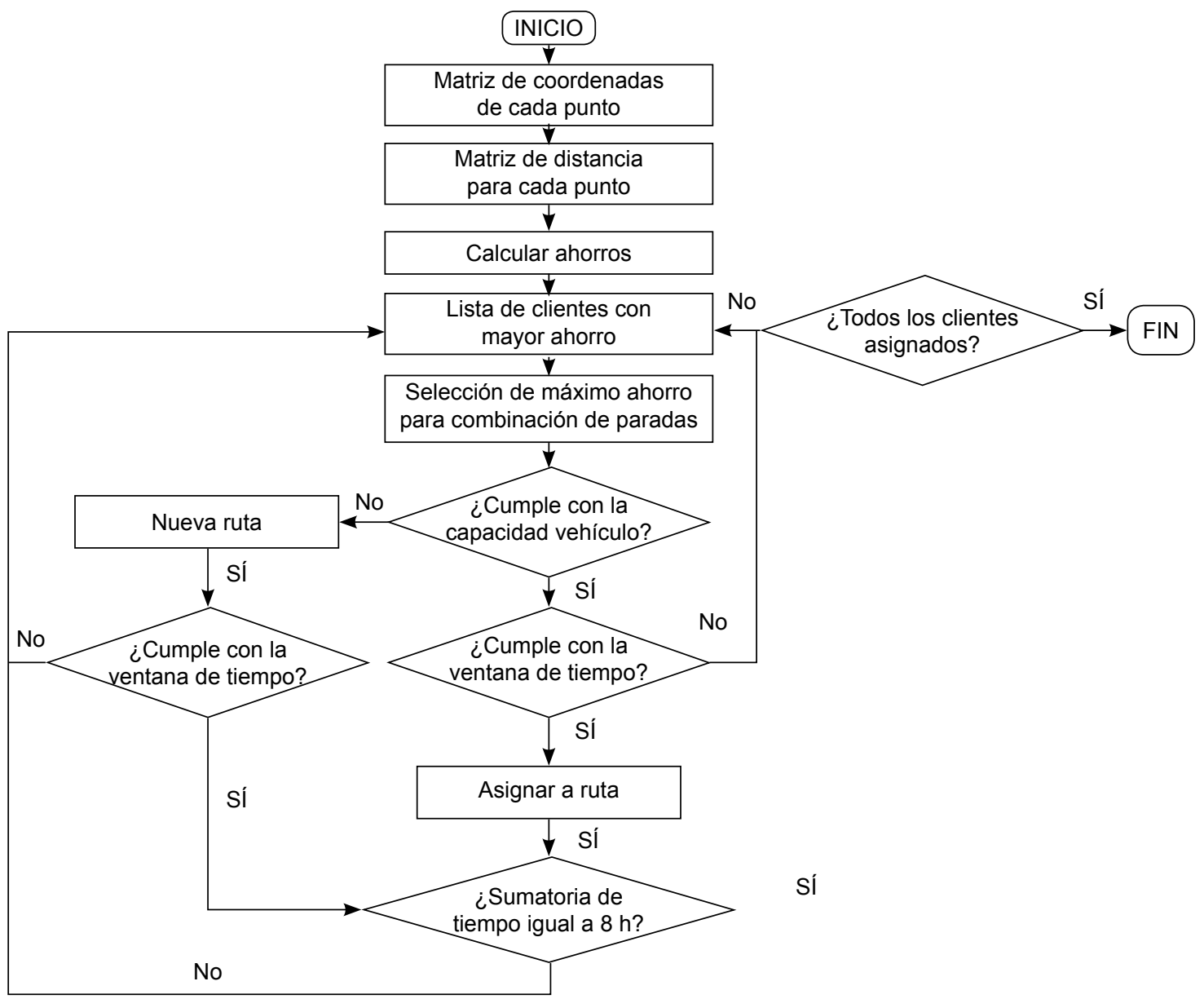

Figura 3. Aplicación método de ahorros con ventanas de tiempo.

\section{RESULTADOS Y DISCUSIÓN}

\section{Análisis situación actual}

La comercializadora del caso de estudio es una pequeña empresa ubicada en la ciudad de Cali, cuya actividad económica principal es la distribución de productos alimenticios típicos de las regiones de Colombia a supermercados de la región. La empresa tiene un total de treinta y cuatro clientes dispersos geográficamente, con una demanda específica que debe ser atendida por una flota de vehículos compuesta por un camión tipo NHR de tres toneladas, con capacidad en volumen de ochenta y cuatro canastillas, y un vehículo de una tonelada, con capacidad en volumen de hasta quince canastillas. Ambos vehículos, una vez finalizadas las labores de distribución, deben regresar al depósito.

En la tabla 1 se presenta la situación actual de distribución por parte de la comercializadora, en la que se observa que para un periodo semanal se realizan un total de siete rutas, cada una de ellas con su respectivo vehículo e información relevante, como distancia total recorrida, tiempo en ruta, porcentaje de utilización y costos. Gracias a este análisis es posible inferir que el común denominador para la situación actual de la empresa es la poca utilización de un vehículo en relación a su capacidad y la subutilización del otro, lo que implica altos costos de operatividad. En la tabla 1 y la 
figura 2 se evidencia que la mayoría de las rutas establecidas por la comercializadora no cumplen con los principios mínimos de una adecuada programación y un óptimo diseño de rutas, como, por ejemplo, "la secuencia de las paradas en una ruta debería formara una figura de una lagrima o que las rutas más eficientes se construyen usando los vehículos más grandes disponibles”(Ballou, 2004, p. 237), con el fin de utilizar su máxima capacidad permitida.

Tabla 1. Resultados obtenidos de la situación actual

\begin{tabular}{cllccc}
\hline Ruta & $\begin{array}{c}\text { Tipo de } \\
\text { vehículo }\end{array}$ & Día de ruta & $\begin{array}{c}\text { Distancia total } \\
\text { recorrida } \mathbf{( k m )}\end{array}$ & $\begin{array}{c}\text { \% promedio de } \\
\text { utilización del vehículo }\end{array}$ & Costos totales (\$) \\
\hline 1 & Camión & Lunes & 53.83 & $16.66 \%$ & $\$ 113827$ \\
2 & Camión & Martes & 35.43 & $5.95 \%$ & $\$ 53873$ \\
3 & Camioneta & & 62.50 & $140.00 \%$ & $\$ 100551$ \\
4 & Camión & Miércoles & 54.53 & $51.19 \%$ & $\$ 140132$ \\
5 & Camión & Jueves & 35.00 & $38.10 \%$ & $\$ 131685$ \\
6 & Camioneta & & 60.75 & $120.00 \%$ & $\$ 96155$ \\
7 & Camión & Viernes & 71.09 & $15.48 \%$ & $\$ 95456$ \\
\hline
\end{tabular}

Los resultados mostrados en la tabla 1 fueron obtenidos del Departamento Administrador de Control de Transporte (DACT), sistema encargado de llevar la información operacional de las rutas que actualmente se manejan, así como el número de vehículos, rutas y distancias totales. El promedio de utilización y costos totales fueron calculados. Los costos totales se calcularon a partir de la estructura de costos de operación vehicular para transporte de carga del Ministerio de Transporte de Colombia.

La figura 4 representa las rutas generadas por la situación actual de la comercializadora de distribución, según características descritas anteriormente:

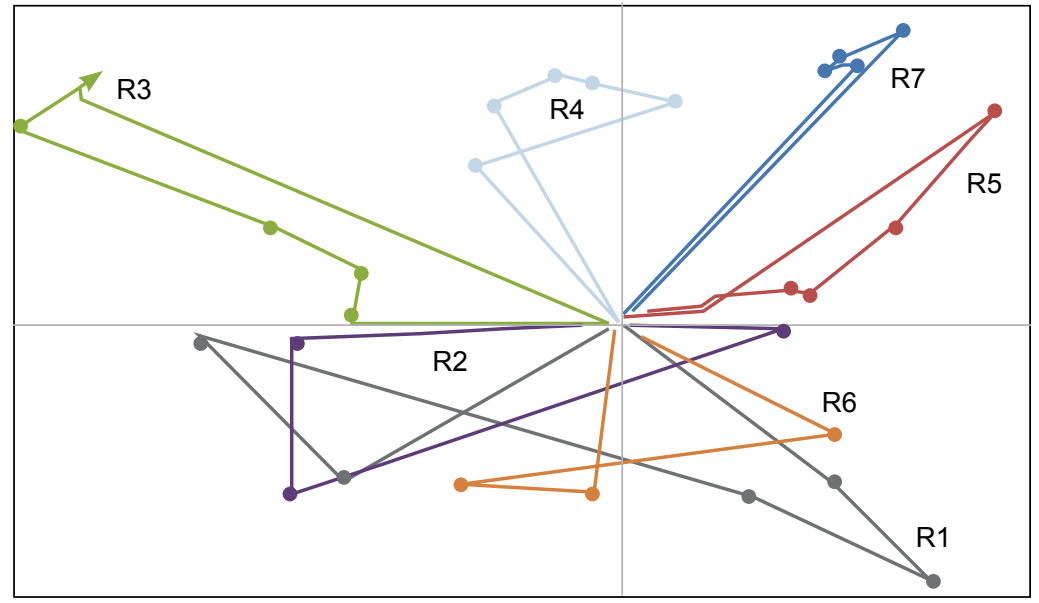

Figura 4. Rutas generadas por la metodología actual de la comercializadora

\section{Descripción del modelo}

Una vez conocidas las características prácticas del problema, se realiza una revisión exhaustiva de la literatura existente, con el fin de seleccionar un modelo matemático que aborde estas caracterís- 
ticas, para posteriormente adaptarlo y realizar pruebas a través de un servidor que permita solucionarlo y así controlar y ajustarlo y luego extraer e interpretarla información. Finalmente, el modelo seleccionado fue el presentado por Toth y Vigo (2014). Se seleccionó este modelo principalmente por la flexibilidad en cuanto al manejo de amplios rangos de restricciones prácticas, como las planteadas por el caso de estudio. Para el presente problema se han definido los siguientes supuestos: i) existe un único depósito y cada vehículo inicia y finaliza en él; ii) la flota de vehículos es heterogénea (capacidades distintas) y se debe considerar sus respectivas capacidades; iii) cada vehículo realiza un único viaje; iv) el costo de transporte está asociado a la distancia total transcurrida; v) se debe visitar a cada cliente al menos una vez; vi) la demanda de cada cliente es conocida, no presenta cambios en el periodo contemplado y debe ser atendida en su totalidad por un solo vehículo; vii) al momento de ejecutar el servicio, solo se realiza labores de entrega de artículos, y viii) se debe considerar ventanas de tiempo para atender a los clientes.

El modelo seleccionado representa al centro de distribución a través dos vértices, 0 y $(n+1)$, el cual no posee demandas y tiempos de servicios definidos. Los clientes son representados por i y j, donde $\mathrm{i}$ difiere de $\mathrm{j}(\mathrm{i} \neq \mathrm{j})$, y en el que cada uno tiene una demanda conocida, como también unas ventanas de tiempo. A continuación se presenta la notación usada en el problema a través de un modelo seleccionado:

Nodos: conjunto de nodos (unión entre puntos de paradas y de depósito) indexados en i y j

Vehículos: conjunto de vehículos disponibles para atender la demanda indexados en $\mathrm{k}$

$\mathrm{I}=$ nodo de partida $\mathrm{i}(1,2, \ldots, 34)$

$\mathrm{J}=$ nodo de llegada $\mathrm{j}(1,2, \ldots, 34)$

$\mathrm{K}=$ vehículos disponibles

Parámetros

$C_{k}$ : costo asociado a la operación del vehículo k

$t_{i:}$ tiempo de viaje para cada arco $\mathrm{A} \in(\mathrm{i}, \mathrm{j})$

$Q_{k}$ : capacidad de cada vehículo

$d_{i}$ : demanda del nodo i

$S_{i}^{i}$ : tiempo de duración del servicio a cada cliente

$a_{i}$ : tiempo más cercano de inicio de servicio en el nodo i

$b_{i}$ : tiempo más lejano de inicio de servicio en el nodo i

$E_{i}$ : representa el tiempo más tardío en el que se puede salir del depósito $(0)$ o $(\mathrm{n}+1)$

$L$ : representa el tiempo más temprano en el que se puede llegar al depósito $(0)$ o $(n+1)$

\section{Variable de decisión}

$X_{i j k}: 1$ si se asigna el vehículo k para recorrer el arco del nodo i al nodo j (de lo contrario, 0 )

$Y_{k}: 1$, si el vehículo $\mathrm{k}$ se asigna a operar una ruta (de lo contrario, 0 )

$W_{i k}$ tiempo de inicio del servicio en el nodo i en el vehículo $\mathrm{k}$

\section{Función objetivo}

Minimizar:

1. $\sum_{k}^{\text {Vehiculos }} \sum_{i}^{\text {NODOS }} \sum_{j}^{\Delta+(i)} C_{k} t_{i j} X_{i j k}$ 
Sujeto a:

2. $\sum_{k}^{\text {Vehiculos }} \sum_{j}^{\Delta+(i)} X_{i j k}=1 ; \forall i \in$ nodos

3. $\sum_{j}^{\Delta+(0)} X_{0 j k}=1 ; \forall k \in$ vehículos

4. $\sum_{i}^{-(i)} X_{i j k} \sum_{i}^{+i} X_{j i k}=0 ; j$ nodos, $k$ vehículos

5. $\sum_{i}^{\Delta-(n+1)} X_{i, n+1, k}=1 ; \forall k \in$ vehículos

6. $W_{i k}+S_{i}+t_{i j}-W_{j k} \leq\left(1-X_{i j k}\right) M ; i \in$ nodos, $j \in$ nodos, $\forall k \in$ vehículos

7. $W_{i k} \geq \sum_{j}^{\Delta-(i)} a_{i} x_{j i k} ; \forall i \in$ nodos, $\forall k \in$ vehículos

8. $W_{i k} \leq \sum_{j}^{\Delta-(i)} b_{i} x_{j i k} ; \forall i \in$ nodos, $\forall k \in$ vehículos

9. $\sum_{i}^{\text {Nodos }} \sum_{j}^{\Delta+(i)} d_{i} x_{i j k} \leq Q_{k}$

10. $\sum_{i}^{\text {Nodos }} \sum_{j}^{\text {Nodos }} X_{i j k} \leq Y_{k} ; \forall k \in$ vehículos

11. $X_{i j k} \in\{0,1\} ; \forall \mathrm{k} \in$ vehículos, $\mathrm{i} \in$ nodos, $\mathrm{j} \in$ nodos

12. $y_{i} \in\{0,1\} ; \forall \mathrm{k} \in$ vehículos, $\mathrm{i} \in$ nodos, $\mathrm{j} \in$ nodos

13. $W_{i k} \geq 0 ; \forall \mathrm{k} \in$ vehículos, $\mathrm{i} \in$ nodos

La restricción 2 garantiza que cada nodo o cliente se asigne a una ruta exactamente. La restricción 3-5 define una ruta de origen-destino para cada vehículo. La restricción 3 establece un origen para cada ruta. La restricción 4 define que el número de arcos entrando a un nodo es igual al número de arcos saliendo de ese nodo. La restricción 5 asegura un destino final en cada ruta. Las restricciones 6-8 consideran la factibilidad de cumplimiento de la demanda impuesta por los nodos clientes en consideración con las ventanas de tiempo asignadas y, asimismo, de la cantidad de producto a enviar: la restricción 6 regula el inicio del servicio para un nodo con respecto al tiempo de inicio del nodo previamente atendido, su tiempo de procesamiento y la distancia en tiempo entre los dos nodos, y las restricciones 7-8 aseguran que los tiempos de inicio del servicio se encuentren dentro del rango permitido por el cliente. La restricción 9 regula la demanda atendida 
en los nodos clientes de cada ruta con respecto a la capacidad de cada vehículo. La restricción 10 asegura la asignación de nodos a un vehículos o los i el vehículo es habilitado para circular. Las restricciones11-13 son restricciones lógicas con respectos a las variables.

\section{Resultados computacionales}

Una vez adaptado el modelo matemático, es implementado en el lenguaje AMPL para realizar las pruebas computacionales ejecutadas en un computador servidor de NEOS SOLVERS con las siguientes características: CPU 2x Intel Xeon E5-2430 (2.2GHz) (12 núcleos en total), HT habilitado, memoria RAM de 64 GB, 2 discos SATA de 2 TB instalados en RAID 1 y red 1 $\mathrm{Gb} / \mathrm{s}$ Ethernet.

Con las pruebas computacionales se obtuvieron los siguientes resultados: para una instancia de 15 clientes, siendo este el número máximo de puntos permitidos por el mismo, donde el programa elimina 350 restricciones y 190 variables, ajustando el problema a 2890 variables distribuidas en 2710 variables binarias y 180 variables lineales, así como un total de 2605 restricciones, todas líneas representadas en 195 restricciones de igualdad y 2410 restricciones de desigualdad, para un total de 360226 iteraciones, 12912 nodos ramificados y enlazados y un objetivo lineal para una solución entera óptima encontrada en referencia al costo de $\$ 47749$.

En la tabla 2 se evidencia los resultados entregados por el NEOS SOLVERS en referencia a la ruta óptima, considerando las características y supuestos establecidos con anterioridad.

Tabla 2. Resultados del NEOS SOLVERS

\begin{tabular}{lcccccc}
\hline Ruta establecida & Vehículo & $\begin{array}{c}\text { Capacidad } \\
\text { utilizada }\end{array}$ & $\begin{array}{c}\% \text { de } \\
\text { utilización }\end{array}$ & $\begin{array}{c}\text { Tiempo de } \\
\text { recorrido }\end{array}$ & Costo & $\begin{array}{c}\text { Función } \\
\text { objetivo }\end{array}$ \\
\hline $\begin{array}{l}\text { 0-7-13-15-12-6-5- } \\
3-9-35\end{array}$ & NHR & 59 & $70 \%$ & 1.21 & $\$ 25909$ & \\
& NHR & 54 & $64 \%$ & 1.01 & $\$ 21626$ & \\
\hline Total costos & & & & & $\$ 47535$ & $\$ 47749$ \\
\hline
\end{tabular}

De acuerdo a los resultados obtenidos en la tabla 4, el servidor arroja 2 rutas, ambas atendidas por el vehículo tipo camión NHR, con porcentajes de utilización en cuanto a capacidad del $70 \%$ para la ruta 1 y del $64 \%$ para la ruta 2, con tiempos totales de recorrido en horas de 1.21 y 1.01 , respectivamente. De igual manera, el servidor entrega el costo para cada ruta, para la cual la suma de ambos costos (cuyo valores en pesos \$47535) concuerda con el costo de la función objetivo (\$47 749.2). El tiempo computacional del servidor fue de 3 horas con 47 minutos y 52 segundos. El modelo matemático establecido y adaptado al problema bajo cuestión cumple con las características prácticas de las tareas de distribución por parte de la comercializadora, principalmente porque considera factores claves como la capacidad de cada vehículo y ventanas de tiempo. La figura 5 ilustra las rutas generadas por el NEOS SOLVERS. 


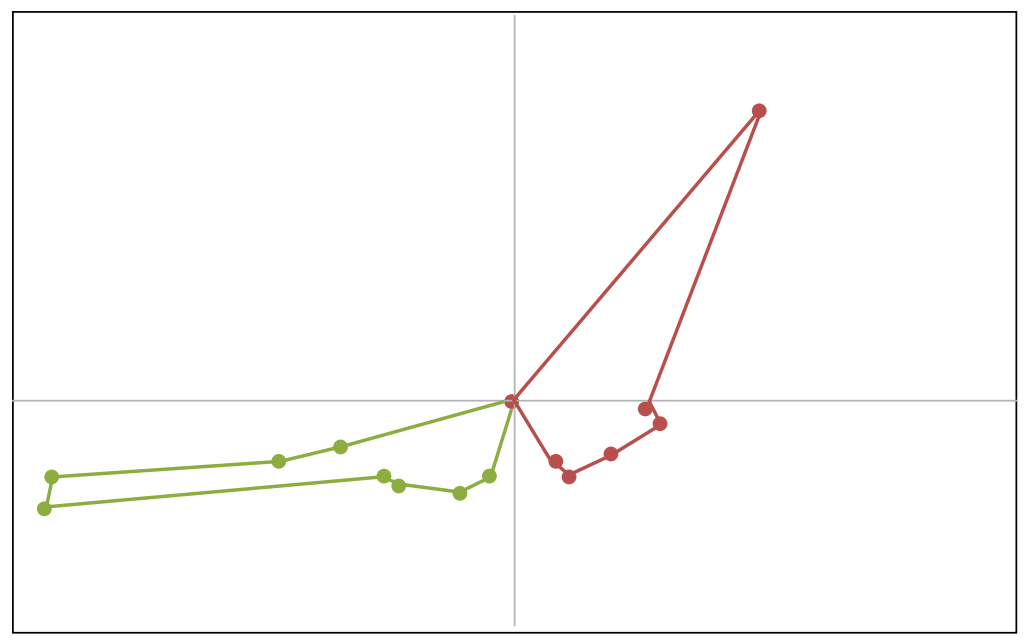

Figura 5. Rutas generadas por NEOS SOLVERS

\section{Resultados heurística}

La selección del método heurístico se realizó a través de una técnica multicriterio AHP (o PAH, por sus siglas en inglés):

Usada para la toma de decisiones con atributos múltiples, permitiendo la descomposición de un problema en una jerarquía ya segura que tanto los aspectos cualitativos como cuantitativos de un problema sean incorporados en el proceso de evaluación, durante la cual la opinión es extraída sistemáticamente por medio de comparaciones entre pares. (González y Bermúdez citados en Saaty, 1987)

Los criterios de selección fueron: flexibilidad para manejar amplios rangos de restricciones prácticas, rapidez en el cálculo de una computadora para problemas con número moderado de paradas y capacidad de generar soluciones que están cerca del óptimo tomados de (Ballou, 2004, p. 263). Las alternativas de decisión son algoritmo de ahorros, heurística de barrido, heurística de inserción y método de asignar primero y rutear después.

La tabla 3 y 4 presentan los resultados de la evaluación de criterios y la matriz de decisión.

Tabla 3. Resultados evaluación de criterios

\begin{tabular}{cr}
\hline \multicolumn{2}{c}{ Vector prioridad } \\
\hline A & 0.703 \\
B & 0.182 \\
C & 0.115 \\
\hline
\end{tabular}

Tabla 4. Resultados matrices de decisión

\begin{tabular}{cc}
\hline \multicolumn{2}{c}{ Matriz de decisión } \\
\hline $\mathrm{a}$ & 0.52 \\
$\mathrm{~b}$ & 0.12 \\
$\mathrm{c}$ & 0.24 \\
$\mathrm{~d}$ & 0.12 \\
\hline Total & 1
\end{tabular}


En la tabla 3 la evaluación de criterios se obtiene a través de la aplicación de una guía de comparación de criterios aplicada a personas expertas en el tema, en la que se evaluó los criterios mencionados para la selección de un método heurístico. En la tabla 4 esta evaluación se obtiene a partir de la consulta de revistas de divulgación científica y libros de divulgación de investigación para comparar las diferentes características entre los métodos heurísticos.

Con base en los resultados se determinó que el método a utilizar es el algoritmo de ahorros. En este punto hay que tener cuenta tres elementos: flexibilidad para manejar rangos amplios de restricciones prácticas; rapidez "para calcular en una computadora problemas con número moderado de paradas, y capacidad de generar soluciones que están cerca del óptimo" (Ballou, 2004, p. 263). Después de la selección del método de ahorros, se procede a su utilización considerando la información suministrada por la comercializadora.

La tabla 5 muestra los resultados generados por la heurística de los ahorros: se crearon 5 rutas y la distancia promedio total de recorrido fue $124.45 \mathrm{~km}$. Por otra parte, la utilización de la capacidad de los vehículos tuvo un promedio mayor en comparación a la metodología actual de la comercializadora. La figura 6 muestra las rutas que fueron creadas por la solución inicial derivada del método de los ahorros.

Tabla 5. Resultados obtenidos por la heurística de los ahorros

\begin{tabular}{clcccc}
\hline Ruta & $\begin{array}{c}\text { Tipo de } \\
\text { vehículo }\end{array}$ & $\begin{array}{c}\text { Distancia total } \\
\text { recorrida } \mathbf{( k m )}\end{array}$ & $\begin{array}{c}\text { Tiempo total } \\
\text { recorrido (horas) }\end{array}$ & $\begin{array}{c}\text { \% promedio de } \\
\text { utilización del vehículo }\end{array}$ & Costos totales (\$) \\
\hline 1 & NHR & 30.51 & 7.18 & $54.76 \%$ & $\$ 153.740$ \\
2 & NHR & 39.70 & 7.25 & $67.86 \%$ & $\$ 155.131$ \\
3 & NHR & 54.24 & 5.65 & $25.00 \%$ & $\$ 121.065$ \\
4 & D-MAX & 67.07 & 3.12 & $86.66 \%$ & $\$ 51.396$ \\
5 & D-MAX & 51.32 & 3.27 & $86.66 \%$ & $\$ 53.935$ \\
\hline
\end{tabular}

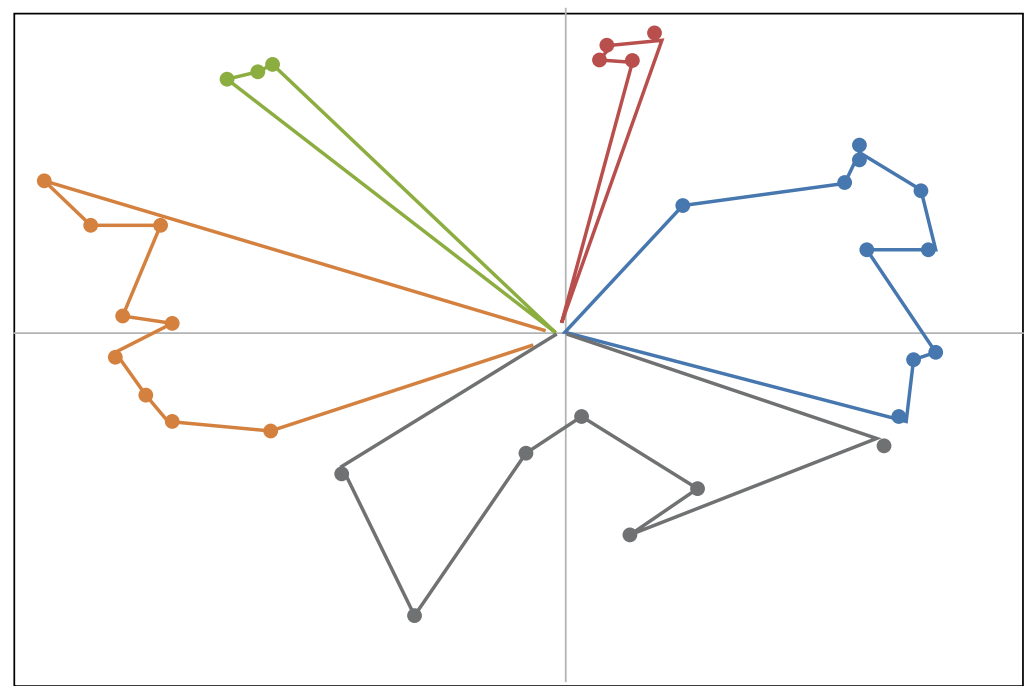

Figura 6. Rutas generadas por la heurística de los ahorros 


\section{Resultados software LOGWARE}

Con el ánimo de comprobar las virtudes de la heurística de los ahorros se utiliza el software LOGWARE y se comparan los resultados de ambas herramientas, evidenciando mejores resultados por parte del método de Clarke y Wright (Ballou, 2004), en cuanto a porcentajes de utilización de los vehículos, distancia recorrida y costos operativos (ver tabla 9).

La aplicación del software generó una solución inicial mejor que la derivada del modelo matemático y que la situación actual de la comercializadora, en relación con el número de rutas, distancia recorrida y costos operativos. La tabla 6 muestra los resultados obtenidos por el LOGWARE.

Tabla 6. Resultados obtenidos por LOGWARE

\begin{tabular}{clcccr}
\hline Ruta & $\begin{array}{c}\text { Tipo de } \\
\text { vehículo }\end{array}$ & $\begin{array}{c}\text { Distancia total } \\
\text { recorrida }(\mathbf{k m})\end{array}$ & $\begin{array}{c}\text { Tiempo total } \\
\text { recorrido (horas) }\end{array}$ & $\begin{array}{c}\text { \% promedio de } \\
\text { utilización del } \\
\text { vehículo }\end{array}$ & Costos totales (\$) \\
\hline 1 & D-MAX & 77.25 & 3.6 & $100.00 \%$ & $\$ 59.342$ \\
2 & NHR & 157.49 & 9.1 & $38.10 \%$ & $\$ 194.851$ \\
3 & NHR & 84.01 & 7.9 & $47.62 \%$ & $\$ 169.156$ \\
4 & NHR & 74.84 & 7.0 & $42.86 \%$ & $\$ 149.885$ \\
5 & D-MAX & 68.98 & 7.3 & $80.00 \%$ & $\$ 120.332$ \\
6 & D-MAX & 71.87 & 7.9 & $100.00 \%$ & $\$ 130.222$ \\
\hline
\end{tabular}

Como sea precia en la tabla 6, LOGWARE creó 6 rutas, el mismo número generado por el método heurístico propuesto. Los otros resultados fueron: $0.09 \mathrm{~s}$ de tiempo computacional, $134.48 \mathrm{~km}$ de distancia total recorrida obtenida y $42.87 \%$ de capacidad de los vehículos. Las rutas generadas por la solución inicial de LOGWARE se muestran en la figura 7.

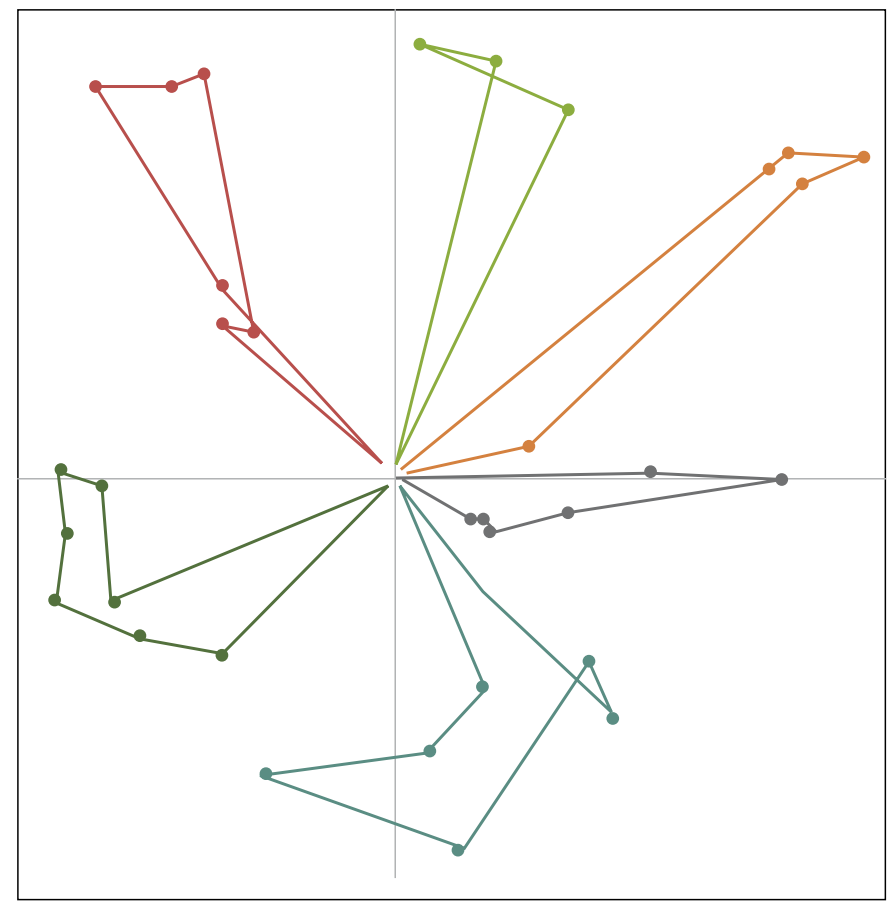

Figura 7. Rutas Generadas por el software LOGWARE 
En las tablas 7 y 8 se presenta la comparación de los valores obtenidos por la situación actual de ruteo de la comercializadora y la heurística de ahorros propuesta, con el fin de analizar e interpretar la información relacionada con el camión tipo NHR y la camioneta D-MAX.

Tabla 7. Comparación de las soluciones obtenidas por la heurística propuesta y la situación actual para el camión NHR

\begin{tabular}{lcccc}
\hline \multicolumn{1}{c}{ Descripción } & $\begin{array}{c}\text { Número } \\
\text { de rutas }\end{array}$ & $\begin{array}{c}\text { Distancia total } \\
\text { recorrida }(\mathbf{k m})\end{array}$ & $\begin{array}{c}\text { \%promedio de } \\
\text { utilización del vehículo }\end{array}$ & \multicolumn{2}{c}{ Costos totales } \\
(\$)
\end{tabular}

Tabla 8. Comparación de las soluciones obtenidas por la heurística propuesta y la situación actual para la camioneta D- MAX

\begin{tabular}{lcccc}
\hline \multicolumn{1}{c}{ Descripción } & $\begin{array}{c}\text { Número de } \\
\text { rutas }\end{array}$ & $\begin{array}{c}\text { Distancia total } \\
\text { recorrida } \mathbf{( k m )}\end{array}$ & $\begin{array}{c}\text { \% promedio de } \\
\text { utilización del vehículo }\end{array}$ & Costos totales (\$) \\
\hline $\begin{array}{l}\text { Solución actual } \\
\text { Heurística de ahorros }\end{array}$ & 2 & 123.25 & $80 \%$ & $\$ 196706$ \\
Clarke-Wright & 2 & 118.39 & $87 \%$ & $\$ 105328$ \\
\hline Porcentaje de mejora & $0 \%$ & $3.94 \%$ & $8.75 \%$ & $46 \%$ \\
\hline
\end{tabular}

Analizando la información suministrada por las tablas 7 y 8, la mejora en cuanto a la reducción de la distancia recorrida, porcentaje de utilización y reducción de costos para ambos vehículos es significativa en comparación a la situación actual de ruteo de la comercializadora, lo que corrobora que la heurística de los ahorros de Clarke-Wright es una alternativa válida para este caso.

En las tablas 9 y 10 se presenta la comparación de los valores obtenidos por la heurística de los ahorros propuesta y el software LOGWARE, en la que se evidencia que si bien ambas aplican el mismo concepto, es posible analizar e interpretar posibles similitudes o diferencias que puedan originarse.

Tabla 9. Comparación de las soluciones obtenidas por la heurística propuesta y el software LOGWARE para el camión NHR

\begin{tabular}{lcccc}
\hline \multicolumn{1}{c}{ Descripción } & $\begin{array}{c}\text { Número } \\
\text { de rutas }\end{array}$ & $\begin{array}{c}\text { Distancia total } \\
\text { recorrida } \mathbf{( k m )}\end{array}$ & $\begin{array}{c}\text { \% promedio de } \\
\text { utilización del vehículo }\end{array}$ & $\begin{array}{c}\text { Costos totales } \\
\mathbf{( \$ )}\end{array}$ \\
\hline Software LOGWARE & 3 & 134.48 & $42.87 \%$ & $\$ 513888$ \\
Heurística de ahorros Clarke-Wright & 3 & 124.45 & $49.33 \%$ & $\$ 429831$ \\
\hline Porcentaje de mejora & $0 \%$ & $7.46 \%$ & $15.08 \%$ & $16.36 \%$ \\
\hline
\end{tabular}

Tabla 10. Comparación de las soluciones obtenidas por la heurística propuesta y el software LOGWARE para la camioneta D-MAX

\begin{tabular}{lcccc}
\hline \multicolumn{1}{c}{ Descripción } & $\begin{array}{c}\text { Número de } \\
\text { rutas }\end{array}$ & $\begin{array}{c}\text { Distancia total } \\
\text { recorrida } \mathbf{( k m )}\end{array}$ & $\begin{array}{c}\text { \% promedio de } \\
\text { utilización del vehículo }\end{array}$ & Costos totales (\$) \\
\hline $\begin{array}{l}\text { Software LOGWARE } \\
\text { Heurística de ahorros }\end{array}$ & 3 & 568.09 & $93.33 \%$ & $\$ 309899$ \\
Clarke-Wright & 2 & 118.39 & $87 \%$ & $\$ 105328$ \\
\hline Porcentaje de mejora & $33 \%$ & $79.16 \%$ & $6.78 \%$ & $66 \%$ \\
\hline
\end{tabular}


Como se observa en la tabla 9 y 10, al igual que el análisis para la situación actual de la comercializadora con la heurística de ahorros propuesta, esta última presenta mejoras significativas que están relacionadas con factores relevantes, como la reducción de la distancia total recorrida y de los costos para ambos vehículos, así como también en la reducción de la utilización de la flota vehicular. En este orden de ideas, si consideramos los resultados expuestos, la mejor opción para el caso de estudio es la heurística de los ahorros de Clarke-Wright, ya que cumple con la función objetivo del modelo.

\section{Conclusiones}

En el presente artículo se propone mediante el uso de la heurística de los ahorros de ClarkeWright la solución a un problema de ruteo de vehículos con ventanas de tiempo y flota heterogénea. En particular, la heurística propuesta mejora la actividad de ruteo de una empresa pequeña, al reducir la distancia total recorrida por todos los vehículos y, de forma indirecta, al minimizar el número de vehículos requeridos para ocuparse de todas las paradas. El resultado de la heurística es un valor de ahorro, seleccionado para la combinación de puntos de cada ruta establecida, que disminuye de manera eficiente el costo de transporte aplicando este método.

La presentación del caso, aplicado a una pequeña empresa colombiana encargada de la distribución de alimentos, permite visibilizar las grandes oportunidades de mejora en los niveles de competitividad que representa este tipo de herramientas para las PyME de nuestro país; mejoras ligadas a la reducción de costos operativos de transporte, distribución y servicio al cliente.

Otras investigaciones que adopten esta perspectiva podrían estar relacionados con el uso de procedimientos metaheurísticos en problemas similares al planteado en el presente artículo y la adaptación del modelo para que tenga en cuenta el servicio al cliente y el impacto financiero.

\section{RefERENCIAS}

Ballou, R. (2004). Logística Administración de la cadena de suministro (5ª ed.). Naucalpan de Juárez, México: Pearson Educación.

Belfiore, P., y Yoshizaki, H. (2013).Heuristic methods for the fleet size and mix vehicle routing problem with time windows and split deliveries. Computers and Industrial Engineering, 64(2), 589-601. https://doi.org/10.1016/j.cie.2012.11.007

Ciancio, C., Laganá, D., y Vocaturo, F. (2018). Branch-price-and-cut for the mixed capacitated general routing problem with time windows. European Journal of Operational Research, 267(1), 187-199. https://doi.org/10.1016/j.ejor.2017.11.039

Domínguez-Martín, B., Rodríguez-Martín, I. y Salazar-González, J. (2018). The driver and vehicle routing problem. Computers and Operations Research, 92, 56-64. https://doi.org/10.1016/j. cor.2017.12.010

El-Sherbeny, N. (2010). Vehicle routing with time windows: an overview of exact ,heuristic and metaheuristic methods. Journal of King Saud University-Science, 22(3), 123-131. https://doi. org/10.1016/j.jksus.2010.03.002

González, G., y González, F. (2006). Metaheurísticas aplicadas al ruteo de vehículos. Un caso de estudio. Parte 1 : Metaheuristics applied to vehicle routing. A case study. Revista Ingeniería e Investigación, 26(3), 149-156. 
Golden, B., Assad, A., Levy, L, y Gheysens, F. (1984).The fleet size and mix vehicle routing problem. Computers and Operations Research, 11(1), 49-66 https://doi.org/10.1016/03050548(84)90007-8

Hiermann, G., Puchinger, J., Ropke, S., y Hartl, R. (2015). The electric fleet size and mix vehicle routing problem with time windows and recharging stations. European Journal of Operational Research, 252(3), 995-1018. https://doi.org/10.1016/j.ejor.2016.01.038

Liu, F., y Shen, S. (1999). The fleet size and mix vehicle routing problem with time windows. The Journal of the Operational Research Society, 50(7), 721-732. https://doi.org/10.2307/3010326

Rocha, L., González, E., y Orjuela,J. (2011). Una revisión al estado del arte del problema de ruteo de vehículos: evolución histórica y métodos de solución. Ingeniería, 16(2), 35-55.

Olivera, A. (2004). Heurísticas para problemas de ruteo de vehiculos. Recuperado de https://www. fing.edu.uy/inco/pedeciba/bibliote/reptec/TR0408.pdf

Rocha, L., González, El, y Orjuela, A.(2011).Una Revisión al estado del arte del problema de ruteo de vehículos: evolución histórica y métodos de solución. Ingeniería, 16(2), 35-55.

Paraskevopoulos, D., Repoussis, P., Tarantilis, C., Ioannou, G., y Prastacos, G. (2008). Reactive variable neighborhood tabu search for heterogeneous fleet VRP and scheduling. Journal ofHeuristics, 14(5), 425-455.

Repoussis, P, y Tarantilis, C. (2010). Solving the fleet size and mix vehicle routing problem with time windows via adaptive memory programming. Transportation Research Part C: Emerging Technologies, 18(5), 695-712. https://doi.org/10.1016/j.trc.2009.08.004

Saaty, R. (1987). The analytic hierarchy process-what its and how its used. Mathematical Modelling, 9(3-5), 161-176. https://doi.org/10.1016/0270255 (87)90473-8

Salhi, S., Wassan, N., y Hajarat, M. (2013). The fleet size and mix vehicle routing problem with backhauls: formulation and set partitioning-based heuristics. Transportation Research Part E: Logistics and Transportation Review, 56, 22-35 https://doi.org/10.1016/j.tre.2013.05.005

Sepúlveda,J., Escobar, J., y Adarme-Jaimes, W. (2014).An algorithm for the routing problem with split deliveries and time windows (SDVRPTW) applied on retail SME distribution activities. Dyna, 81(187), 223-231. https://doi.org/10.15446/dyna.v81n187.46104

Solomon, M. (1987). Algorithms for the vehicle routing and scheduling problems with time window constraints. Operations Research, 35(2), 254-265. https://doi.org/10.1287/opre.35.2.254

Toro, O., Escobar, Z., y Granada, E. (2016). Literature review on the vehicle routing problem in the green transportation context. Luna Azul,42,362-387.https://doi.org/10.17151/luaz.2016.42.21

Toth, P., y Vigo, D. (eds.). (2014).Vehicle routing problem, methods, and application (2a edición). Philadelphia, EE. UU.: Society for Industrial and Applied Mathematics. 
